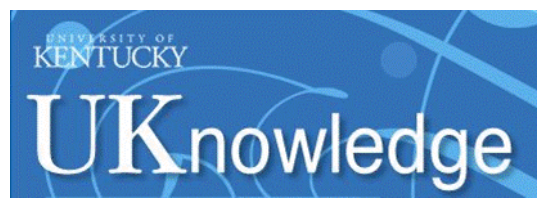

University of Kentucky

UKnowledge

$1-1-2007$

\title{
Mid-Infrared Galaxy Classification Based on Silicate Obscuration and PAH Equivalent Width
}

\author{
H. W. W. Spoon \\ Cornell University
}

J. A. Marshall

Cornell University

J. R. Houck

Cornell University

Moshe Elitzur

University of Kentucky, moshe@pa.uky.edu

L. Hao

Cornell University

See next page for additional authors

Follow this and additional works at: https://uknowledge.uky.edu/physastron_facpub

Part of the Astrophysics and Astronomy Commons, and the Physics Commons

Right click to open a feedback form in a new tab to let us know how this document benefits you.

\section{Repository Citation}

Spoon, H. W. W.; Marshall, J. A.; Houck, J. R.; Elitzur, Moshe; Hao, L.; Armus, L.; Brandl, B. R.; and Charmandaris, V., "Mid-Infrared Galaxy Classification Based on Silicate Obscuration and PAH Equivalent Width" (2007). Physics and Astronomy Faculty Publications. 208.

https://uknowledge.uky.edu/physastron_facpub/208

This Article is brought to you for free and open access by the Physics and Astronomy at UKnowledge. It has been accepted for inclusion in Physics and Astronomy Faculty Publications by an authorized administrator of UKnowledge. For more information, please contact UKnowledge@lsv.uky.edu. 


\section{Mid-Infrared Galaxy Classification Based on Silicate Obscuration and PAH Equivalent Width}

Digital Object Identifier (DOI)
http://dx.doi.org/10.1086/511268

Notes/Citation Information

Published in The Astrophysical Journal Letters, v. 654, no. 1, p. L49-L52.

(C) 2007. The American Astronomical Society. All rights reserved.

The copyright holder has granted permission for posting the article here.

Authors

H. W. W. Spoon, J. A. Marshall, J. R. Houck, Moshe Elitzur, L. Hao, L. Armus, B. R. Brandl, and V. Charmandaris 


\title{
MID-INFRARED GALAXY CLASSIFICATION BASED ON SILICATE OBSCURATION AND PAH EQUIVALENT WIDTH
}

\author{
H. W. W. Spoon, ${ }^{1,2}$ J. A. Marshall,${ }^{1}$ J. R. Houck, ${ }^{1}$ M. Elitzur, ${ }^{3}$ L. Hao, ${ }^{1}$ L. Armus, ${ }^{4}$ \\ B. R. BRAndL, ${ }^{5}$ and V. Charmandaris ${ }^{6,7}$ \\ Received 2006 October 26; accepted 2006 November 14; published 2006 December 18
}

\begin{abstract}
We present a new diagnostic diagram for mid-infrared spectra of infrared galaxies based on the equivalent width of the $6.2 \mu \mathrm{m}$ PAH emission feature and the strength of the $9.7 \mu \mathrm{m}$ silicate feature. Based on the positions in this diagram, we classify galaxies into nine classes ranging from continuum-dominated AGN hot dust spectra and PAHdominated starburst spectra to absorption-dominated spectra of deeply obscured galactic nuclei. We find that galaxies are systematically distributed along two distinct branches: one of AGN and starburst-dominated spectra and one of deeply obscured nuclei and starburst-dominated spectra. The separation into two branches likely reflects a fundamental difference in the dust geometry in the two sets of sources: clumpy versus nonclumpy obscuration. Spectra of ULIRGs are found along the full length of both branches, reflecting the diverse nature of the ULIRG family.
\end{abstract}

Subject headings: galaxies: active — galaxies: ISM — galaxies: starburst — infrared: galaxies

\section{INTRODUCTION}

Over the last decade several diagnostic diagrams have been proposed to quantify the contribution of star formation and active galactic nucleus (AGN) activity to the infrared luminosity of infrared galaxies based on the mid-infrared (to far-infrared) continuum slope, the polycyclic aromatic hydrocarbon (PAH) line-tocontinuum ratio, the PAH to far-infrared luminosity ratio, and the ratio of a high- to a low-ionization forbidden line such as [Ne v]/ [Ne II] (Genzel et al. 1998; Lutz et al. 1998; Laurent et al. 2000; Peeters et al. 2004; Dale et al. 2006; Sturm et al. 2006). However, none of these diagrams takes into account the effects of strong obscuration of the nuclear power source.

With the advent of the Infrared Spectrograph (IRS; Houck et al. 2004) on board the Spitzer Space Telescope (Werner et al. 2004), astronomers have been handed a powerful tool to study the 5-37 $\mu \mathrm{m}$ range for a wide range of galaxy types at an unprecedented sensitivity. This enables for the first time a systematic study of a large number of galaxies over the wavelength range in which amorphous silicate grains have strong opacity peaks due to the $\mathrm{Si}-\mathrm{O}$ stretching and the $\mathrm{O}-\mathrm{Si}-\mathrm{O}$ bending modes centered at 9.7 and $18 \mu \mathrm{m}$, respectively. Here we introduce the strength of the $9.7 \mu \mathrm{m}$ silicate feature as a tool to distinguish between different dust geometries in the central regions of (ultra-)(luminous) infrared galaxies (ULIRGs) as part of a new diagnostic diagram and mid-infrared galaxy classification scheme.

\section{OBSERVATIONS AND DATA REDUCTION}

The results presented in this Letter are based on both Spitzer IRS and ISO SWS observations. The core sample is formed by the IRS Guaranteed Time Observation (GTO) ULIRG sample (PID 105; P.I.: J. R. Houck), which comprises $\sim 100$ ULIRGs in the redshift range $0.02<z<0.93$. This sample is compared to samples of AGN and starburst templates from the

\footnotetext{
${ }^{1}$ Astronomy Department, Cornell University, Ithaca, NY; spoon@astro.cornell.edu. ${ }^{2}$ Spitzer Fellow.

${ }^{3}$ Department of Physics and Astronomy, University of Kentucky, Lexington, KY.

${ }^{4}$ California Institute of Technology, Spitzer Science Center, Pasadena, CA.

${ }^{5}$ Leiden Observatory, Leiden, Netherlands.

${ }^{6}$ Department of Physics, University of Crete, Heraklion, Greece.

${ }^{7}$ IESL/Foundation for Research and Technology-Hellas, Heraklion, Greece; and Chercheur Associé, Observatoire de Paris, Paris, France.
}

IRS GTO programs 14 (Brandl et al. 2006; Weedman et al. 2005) and 96 and to selected AGNs from GTO programs 82 and 86 (Shi et al. 2006). Additional IRGs, LIRGs, and ULIRGs were taken from program 159 (NGC 1377; Roussel et al. 2006) and from Spitzer DDT program 1096. Archival ISO SWS spectra of starburst nuclei were taken from Sturm et al. (2000).

The Spitzer observations were made with the Short-Low (SL) and Long-Low (LL) modules of the IRS. The spectra were extracted from DROOP level images provided by the Spitzer Science Center (34 using pipeline ver. S11.0.2, 131 using pipeline ver. S14.0) and background-subtracted by differencing the firstand second-order apertures. The spectra were calibrated using the IRS standard stars HD $173511(5.2-20.5 \mu \mathrm{m})$ and $\xi$ Dra (20.5-38.5 $\mu \mathrm{m})$. After flux calibration, the orders were stitched to LL order 1, requiring order-to-order scaling adjustments of typically less than $10 \%$.

\section{ANALYSIS}

For all the spectra in our sample we have measured the equivalent width of the $6.2 \mu \mathrm{m}$ PAH emission feature as well as the strength of the $9.7 \mu \mathrm{m}$ silicate feature and plotted the two quantities in a diagnostic diagram (Fig. 1).

The flux in the $6.2 \mu \mathrm{m}$ PAH emission band is measured by integrating the flux above a spline interpolated local continuum from 5.95 to $6.55 \mu \mathrm{m}$. The equivalent width (EW) of the PAH feature is then obtained by dividing the integrated PAH flux by the interpolated continuum flux density below the peak $(\sim 6.22 \mu \mathrm{m})$ of the PAH feature.

The apparent strength of the $9.7 \mu \mathrm{m}$ silicate feature is inferred by adopting a local mid-infrared continuum and evaluating the ratio of observed flux density $\left(f_{\text {obs }}\right)$ to continuum flux density $\left(f_{\text {cont }}\right)$ at $9.7 \mu \mathrm{m}$ and defining

$$
S_{\text {sil }}=\ln \frac{f_{\text {obs }}(9.7 \mu \mathrm{m})}{f_{\text {cont }}(9.7 \mu \mathrm{m})} .
$$

For sources with a silicate absorption feature, $S_{\text {sil }}$ can be interpreted as the negative of the apparent silicate optical depth.

Given the great diversity among our mid-infrared galaxy spectra, there is no "one-size-fits-all" procedure to define the local continuum in all spectra. We therefore developed separate methods for three distinctly different types of mid-infrared gal- 


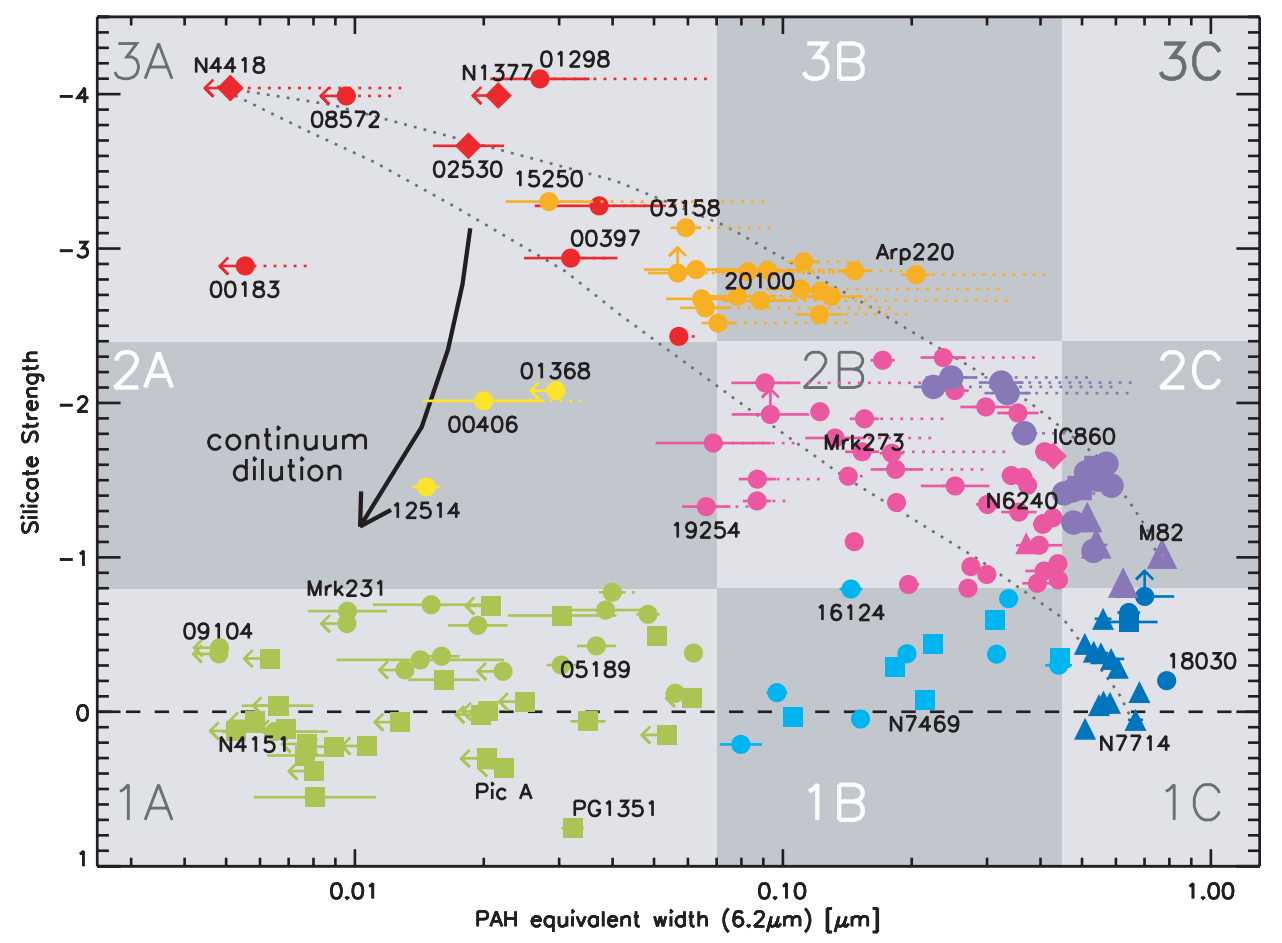

FIG. 1.-Diagnostic plot of the equivalent width of the $6.2 \mu \mathrm{m}$ PAH emission feature vs. the $9.7 \mu \mathrm{m}$ silicate strength. Upper and lower limits are denoted by arrows. The galaxy spectra are classified into nine classes (identified by nine shaded rectangles) based on their position in this plot. Colors are used to distinguish the various classes. From class 1A to 1B, 1C, 2A, 2B, 2C, 3A, and 3B the colors used are green, cyan, dark blue, yellow, pink, purple, red, and orange. After class assignment the PAH equivalent widths were corrected for the effect of $6 \mu \mathrm{m}$ water ice absorption on the $6.2 \mu \mathrm{m}$ continuum. The extent of the individual corrections are indicated by dotted horizontal lines. The two dotted black lines are mixing lines between the spectrum of the deeply obscured nucleus of NGC 4418 and the starburst nuclei of M82 and NGC 7714, respectively. Galaxy types are distinguished by their plotting symbol: Filled circles: ULIRGs and HyLIRGs. Filled triangles: Starburst galaxies. Filled squares: Seyfert galaxies and QSOs. Filled diamonds: Other infrared galaxies.

axy spectra: continuum-dominated spectra, PAH-dominated spectra, and absorption-dominated spectra. These methods are illustrated in Figure 2 and described in its caption. We are forcing the local continuum to touch down at 14.0-14.5 $\mu \mathrm{m}$, because the ISM dust cross section decreases between the two silicate peaks in this region. Detailed radiative transfer calculations verify that our interpolation procedure properly reproduces the emission that would be generated by dust stripped of its silicate features (M. Sirocky et al. 2007, in preparation).

\subsection{Galaxy Classification}

The galaxy spectra in Figure 1 are classified into nine different classes based on their 6.2 $\mu \mathrm{m}$ PAH EW and $9.7 \mu \mathrm{m}$ silicate strength. The parameter space covered by the various classes is indicated by shaded rectangles in Figure 1. Average mid-infrared spectra for the eight populated classes are shown in Figure 3. The average spectra were constructed by normalizing all spectra to unity at $14.5 \mu \mathrm{m}$ flux before the averaging process. In order to maximize the signal-to-noise ratio $(\mathrm{S} / \mathrm{N})$ of the average spectra, low $\mathrm{S} / \mathrm{N}$ spectra were discarded from the process. Below we describe the eight average spectra in the order (bottom to top) they are presented in Figure 3. The ninth class, 3C, is not populated.

The class 1A spectrum is characterized by a nearly featureless hot dust continuum with a very weak silicate absorption feature at $9.7 \mu \mathrm{m}$. The class $1 \mathrm{~B}$ spectrum differs from the class $1 \mathrm{~A}$ spectrum by clearly showing the family of PAH emission features at $6.2,7.7,8.6,11.2,12.7$, and $17.3 \mu \mathrm{m}$ on top of a hot dust continuum. This hot dust continuum is nearly absent in the class $1 \mathrm{C}$ spectrum, allowing the PAH emission features to dominate the mid-infrared spectral appearance. Silicate absorption at $9.7 \mu \mathrm{m}$ becomes noticable in the class $2 \mathrm{C}$ spectrum as an increased depth of the depression between the 6-9 and 11-13 $\mu \mathrm{m}$ PAH emission complexes. Another marked difference with the class $1 \mathrm{C}$ spectrum is the steepening of the $20-30 \mu \mathrm{m}$ continuum and the appearance of an $18 \mu \mathrm{m}$ silicate absorption feature. In the class $2 \mathrm{~B}$ spectrum the PAH features appear weaker than those in the class $2 \mathrm{C}$ spectrum. In addition, the spectrum starts to show a $6 \mu \mathrm{m}$ water ice and a $6.85 \mu \mathrm{m}$ aliphatic hydrocarbon absorption band. In the class $3 \mathrm{~B}$ spectrum these absorption features reach their maximum depths, while the 9.7 and $18 \mu \mathrm{m}$ silicate features continue to increase in depth up to class 3A. Absorption features of crystalline silicates (Spoon et al. 2006) appear in the spectra of classes 3B and $3 \mathrm{~A}$ at 16,19 , and $23 \mu \mathrm{m}$. Equivalent widths of PAH emission features and emission lines decrease from class $2 \mathrm{~B}$ to $3 \mathrm{~B}$ and $3 \mathrm{~A}$. Note especially the change in shape of the $7.7 \mu \mathrm{m}$ PAH feature as it first broadens and then disappears going from class $2 \mathrm{~B}$ to $3 \mathrm{~B}$ and $3 \mathrm{~A}$. Finally, the class $2 \mathrm{~A}$ spectrum differs from the class 3A spectrum mainly by a clearly lower apparent depth of the 9.7 and $18 \mu \mathrm{m}$ silicate features. Note that the spectral structure in individual spectra may differ substantially from the average properties of the classes. In Figure 3 this is represented by the $1 \sigma$ dispersion ranges around the individual average spectra.

\subsection{Galaxy Distribution}

The galaxies shown in Figure 1 are color-coded according to their galaxy classification. However, their positions may differ from their original assignments, as in Figure 1 the $6.2 \mu \mathrm{m}$ PAH EW has been corrected for the effect of $6 \mu \mathrm{m}$ water ice absorption on the $6.2 \mu \mathrm{m}$ continuum through substitution of the observed 


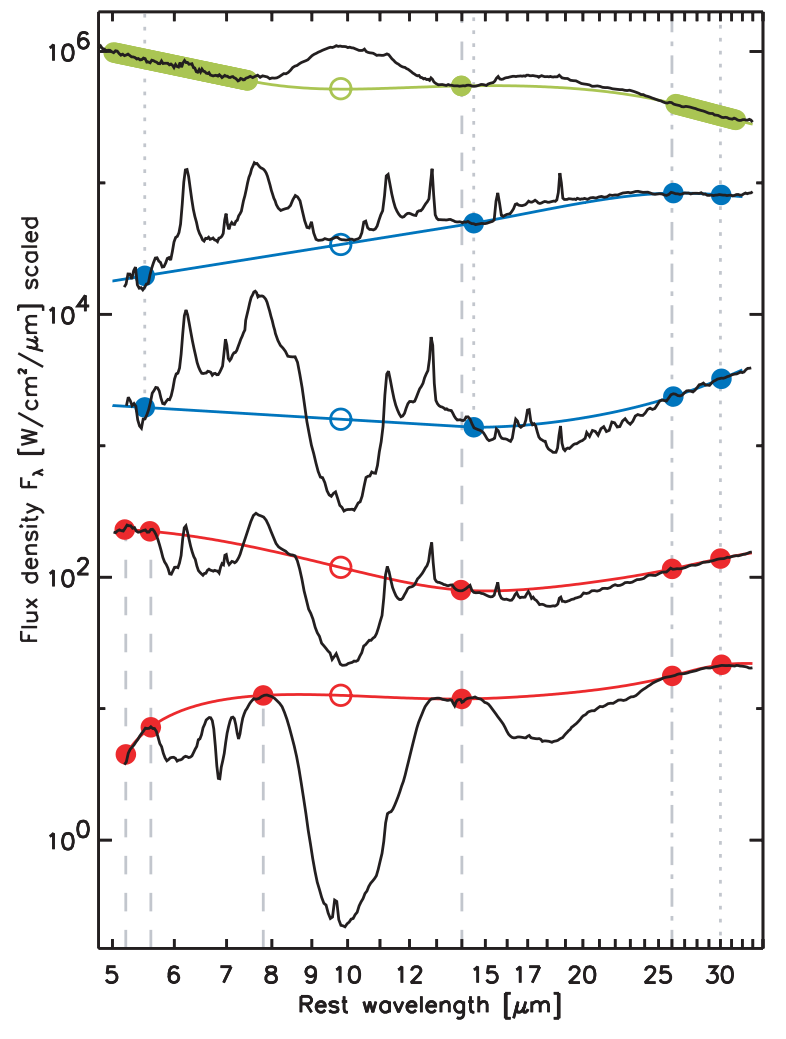

FIG. 2.-Comparison of several methods used to determine the apparent $9.7 \mu \mathrm{m}$ silicate optical depth. The three methods differ by the way the local midinfrared continuum is defined. For continuum-dominated spectra (top) simple logarithmic linear fits are made to the 5.0-7.5 and 26.1-31.5 $\mu \mathrm{m}$ Jy-spectrum. These are spline interpolated over the 7.5-26 $\mu \mathrm{m}$ range, using a continuum point at $14.0 \mu \mathrm{m}$ to guide the spline in the intersilicate-feature range. Pivots and fit are shown in green. For PAH-dominated spectra (2nd and 3rd spectrum) the 5.5$14.5 \mu \mathrm{m}$ continuum is estimated from a power-law interpolation over this range. From 14.5 to $26.0 \mu \mathrm{m}$ this power law is continued as a spline function with a curvature determined by continuum pivots at 26.0 and $30.0 \mu \mathrm{m}$. Pivots and fit are shown in blue. For absorption-dominated spectra (4th and 5th spectrum) the local 5.6-26 $\mu \mathrm{m}$ continuum is determined from a spline interpolation to continuum pivots at 5.2, 5.6, 14.0, and $26.0 \mu \mathrm{m}$. An additional continuum pivot at $7.8 \mu \mathrm{m}$ is included for spectra with very little PAH emission (e.g., bottom spectrum). Pivots and fit are shown in red. In all three cases the $9.7 \mu \mathrm{m}$ silicate strength is computed by evaluating the ratio of observed and interpolated continuum at its peak. From top to bottom, the five spectra shown are the IRS lowresolution spectra of PG 1351+640, NGC 7714, NGC 3628, UGC 5101, and NGC 4418.

$6.2 \mu \mathrm{m}$ continuum by the $6.2 \mu \mathrm{m}$ continuum that was defined to infer the $9.7 \mu \mathrm{m}$ silicate strength. The ice correction is justified assuming that the ice is part of the obscuring medium behind the PAH-emitting region. The correction is applied only to spectra clearly showing the imprint of water ice absorption, as found among the spectra of classes $2 \mathrm{~B}, 2 \mathrm{C}, 3 \mathrm{~A}$, and $3 \mathrm{~B}$. The extent of the correction for individual spectra is indicated in Figure 1 by horizontal dotted lines.

The ULIRGs, Seyfert galaxies, quasars, and starburst galaxies shown in Figure 1 are not distributed randomly through the diagram. Instead, the galaxies appear to be distributed along two branches: one extending horizontally from the positions of the prototypical Seyfert 1 nucleus NGC 4151 (Weedman et al. 2005) to the prototypical starburst nucleus NGC 7714 (Brandl et al. 2004), the other branch extending diagonally from the prototypical deeply obscured nucleus of NGC 4418 (Spoon et al. 2001) to the starburst nuclei of M82 (Sturm et al. 2000) and NGC 7714. Very few sources are found above and to the

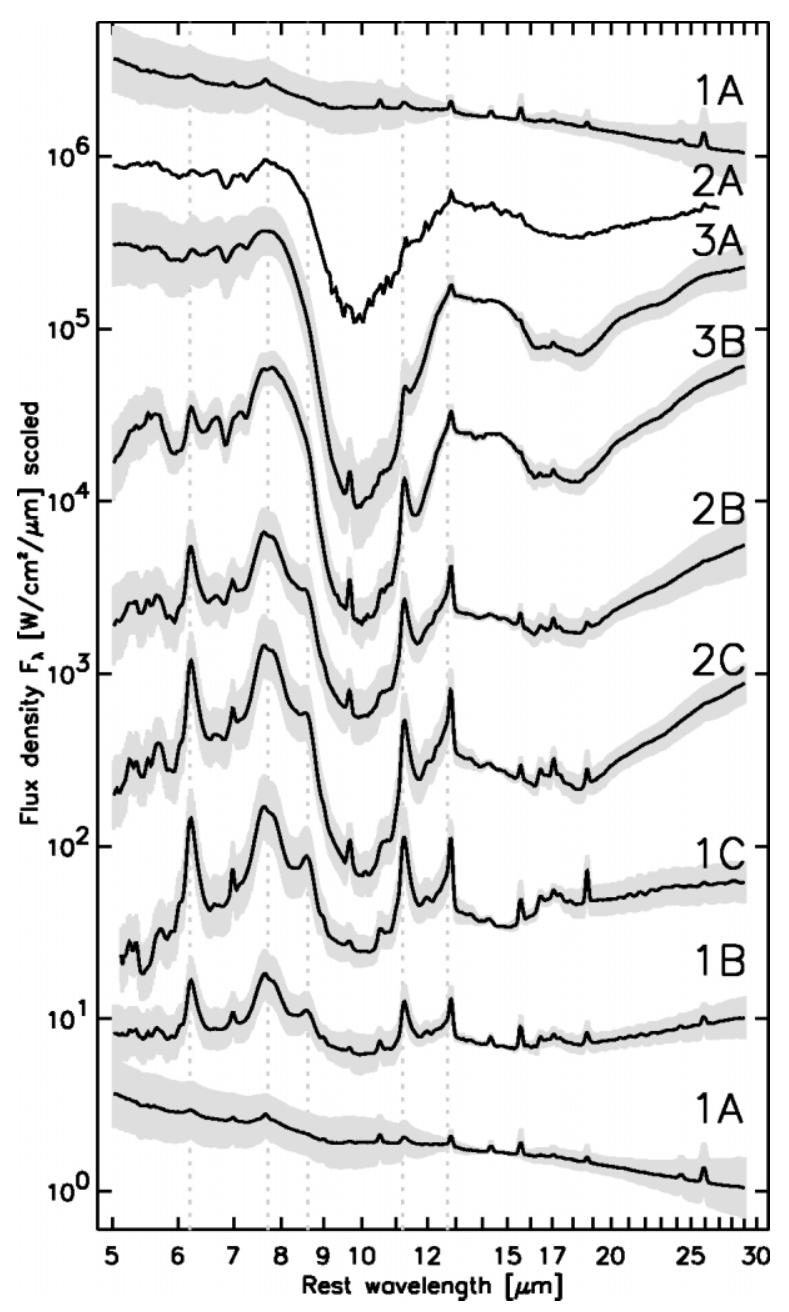

FIG. 3.-Average spectra for eight of the classes of galaxy spectra defined in Fig. 2, along with the $1 \sigma$ dispersion range (gray shading). Most of the dispersion is due to the overall slope of the spectrum. Spectra at the bottom of the figure (classes 1A and 1B) are dominated by hot dust emission, spectra in the middle (classes $1 \mathrm{C}$ and $2 \mathrm{C}$ ) by PAH emission and spectra toward the top by absorption features (classes 3B, 3A, and 2A). Vertical dotted lines denote the central wavelengths of the family of PAH emission features at 6.2, 7.7, 8.6, 11.2, and $12.7 \mu \mathrm{m}$.

right of the diagonal branch and in between the two branches (the class 2A domain).

The three extremes distinguish between AGN-heated hot dust spectra (class 1A), PAH-dominated spectra (classes 1C and 2C), and absorption-dominated spectra (class 3A). The galaxies lined up along the two branches in between the extremes show signatures of both extremes to varying proportions. The galaxies along the horizontal branch may be thought of as combinations of AGN and starburst activity, while the galaxies along the diagonal branch may be thought of as intermediate stages in between a fully obscured galactic nucleus and an unobscured nuclear starburst. The latter is illustrated by the two dotted lines in Figure 1, which represent mixing lines between the spectrum of NGC 4418 on one extreme and either NGC 7714 or M82 on the other. The large majority of galaxies on the diagonal branch fall in between these mixing lines. One notable exception is Arp 220.

Relatively few galaxies are found in the section between the two branches: the domain of the class 2 A sources. Spectroscopically, there are several simple scenarios that will populate this class from adjacent classes. A class $3 \mathrm{~A}$ galaxy can be turned 
into a class $2 \mathrm{~A}$ galaxy by adding a featureless hot dust continuum to the class $3 \mathrm{~A}$ absorbed continuum spectrum. The main effect of this continuum dilution is the filling in of the deep $9.7 \mu \mathrm{m}$ silicate feature, resulting in a less pronounced feature. Increasing the dilution not only further decreases the depth of the silicate feature but also increases the continuum at $6.2 \mu \mathrm{m}$, resulting in both a less negative silicate strength and a lower $6.2 \mu \mathrm{m}$ PAH EW. The effect of this type of continuum dilution is illustrated in Figure 1 by a curved arrow. Alternatively, a class $1 \mathrm{~A}$ galaxy may be turned into class $2 \mathrm{~A}$ galaxy by passing its mid-infrared spectrum through a foreground cold dust screen. The path that a galaxy will take is straight up, since the PAH EW is not affected by cold foreground extinction.

The presence of foreground dust or dust mixed in within the PAH-emitting region may also explain the difference between class $1 \mathrm{C}$ and $2 \mathrm{C}$ starburst galaxies, and the difference between the classification of the prototypical starburst galaxies NGC 7714 and M82 in particular. In the latter case this may be primarily an orientation effect, as the nuclear starburst in NGC 7714 is observed face-on, whereas the starburst ring in M82 is seen under an inclination of $\sim 80^{\circ}$.

Finally, the complete absence of galaxy spectra classified as 3C must imply that strong, pure foreground, cold dust obscuration is not common in starburst nuclei. The obscuring dust instead contributes to the continuum underlying the $6.2 \mu \mathrm{m}$ $\mathrm{PAH}$ feature, reducing its EW.

\section{DISCUSSION AND CONCLUSIONS}

We have constructed a diagnostic diagram of two mid-infrared spectral quantities, the equivalent width of the $6.2 \mu \mathrm{m}$ PAH feature and the apparent $9.7 \mu \mathrm{m}$ silicate strength, for the purpose of classifying infrared galaxies according to their mid-infrared spectral shape (Fig. 1).

The large majority of galaxies $(>90 \%)$ are dispersed around two branches: a horizontal one extending from continuumdominated spectra to PAH-dominated spectra and a diagonal one spanned between absorption-dominated spectra and PAHdominated spectra. Seyfert galaxies and quasars (Fig. 1, squares) are found exclusively on the horizontal branch characterized by the absence of pronounced silicate features. Starburst galaxy spectra (triangles) are concentrated toward the extreme right tip of the two branches, the locus of PAH-dominated spectra. Of the ULIRGs and hyperluminous IRGs (HyLIRGs) in our sample (circles), about a quarter occupy the same space as the AGNs and starbursts in our sample. The large majority, however, are distributed along the diagonal branch, characterized by increasingly apparent silicate absorption and less-pronounced PAH emission features, with deeply obscured galactic nuclei such as NGC 4418 (Spoon et al. 2001) and NGC 1377 (Roussel et al.
2006) as the only non-ULIRG, non-Seyfert end members on this branch. Note that low-metallicity galaxies (not included in this study) would be distributed along the horizontal branch.

The existence of two distinct regimes of apparent silicate absorption, as suggested by the two distinct branches in Figure 1, is intriguing and likely points to clear differences in the nuclear dust distribution between galaxies on the two branches. Clumpy distributions produce only shallow absorption features (Nenkova et al. 2002) and are the likely explanation for the horizontal branch. The deep silicate absorption found on the slanted branch requires the nuclear source to be deeply embedded in a smooth distribution of dust that is both geometrically and optically thick (Levenson et al. 2006). The transition from clumpy geometry to one dominated by a smooth dust distribution is a possible explanation for the sources located between the branches.

The distribution of ULIRG spectra along the full length of both the horizontal and the diagonal branches once again illustrates the diverse nature of the ULIRG family. However, it also raises the issue of ULIRG evolution. For instance, what evolutionary path has taken IRAS $08572+3915$ to its extreme class $3 \mathrm{~A}$ position in which the central power source is deeply buried? Did the interaction start with both nuclei classified as class $1 \mathrm{C} / 2 \mathrm{C}$ starburst galaxies, which then gradually moved up the diagonal branch as the interaction stengthened and more dust accumulated on the remnant nuclei? And how will IRAS $08572+3915$ evolve from there, once the obscuring screen breaks up and the hidden power sources are revealed? In the case of a hidden starburst, the source likely will move back diagonally toward the starburst locus. However, if the dominant power source is an AGN, will the source cross into the sparsely populated class $2 \mathrm{~A}$ regime, evolving directly toward the AGNdominated class $1 \mathrm{~A}$ regime, or will it first undergo a starburst before settling somewhere along the horizontal branch? The sparse population of the class $2 \mathrm{~A}$ domain either indicates that the crossing time is brief-with few ULIRGs caught in transformation-or that deeply obscured ULIRG nuclei mostly evolve to starbursts (first).

In a more extensive paper (H. W. W. Spoon et al. 2007, in preparation) we will present correlations of additional parameters within the diagram (e.g., mid-IR line ratios, $L_{\mathrm{IR}}, L_{\mathrm{PAH}} / L_{\mathrm{IR}}$, absorption features, nuclear separation, and optical classification).

The authors wish to thank Jerónimo Bernard-Salas, Nancy Levenson, Hélène Roussel, Matthew Sirocky, Alexander Tielens, and Dan Weedman for discussions, and Vandana Desai for help with the data reduction. Support for this work was provided by NASA through contract 1257184 issued by the Jet Propulsion Laboratory, California Institute of Technology under NASA contract 1407. H. W. W. S. was supported under this contract through the Spitzer Space Telescope Fellowship Program.

\section{REFERENCES}

Brandl, B. R., et al. 2004, ApJS, 154, 188

$$
\text { 2006, ApJ, 653, } 1129
$$

Dale, D. A., et al. 2006, ApJ, 646, 161

Genzel, R., et al. 1998, ApJ, 498, 579

Houck, J. R., et al. 2004, ApJS, 154, 18

Laurent, O., Mirabel, I. F., Charmandaris, V., Gallais, P., Madden, S. C., Sauvage, M., Vigroux, L., \& Cesarsky, C. 2000, A\&A, 359, 887

Levenson, N. A., Sirocky, N. M., Hao, L., Spoon, H. W. W., Marshall, J. A., Elitzur, M., \& Houck, J. R. 2006, ApJ, 654, L45

Lutz, D., Spoon, H. W. W., Rigopoulou, D., Moorwood, A. F. M., \& Genzel, R. 1998, ApJ, 505, L103
Nenkova, M., Ivezić, Ž., \& Elitzur, M. 2002, ApJ, 570, L9

Peeters, E., Spoon, H. W. W., \& Tielens, A. G. G. M. 2004, A\&A, 613, 986 Roussel, H., et al. 2006, ApJ, 646, 841

Shi, Y., et al. 2006, ApJ, 653, 127

Spoon, H. W. W., Keane, J. V., Tielens, A. G. G. M., Lutz, D., \& Moorwood, A. F. M. 2001, A\&A, 365, L353

Spoon, H. W. W., et al. 2006, ApJ, 638, 759

Sturm, E., Lutz, D., Tran, D., Feuchtgruber, H., Genzel, R., Kunze, D., Moorwood, A. F. M., \& Thornley, M. D. 2000, A\&A, 358, 481

Sturm, E., et al. 2006, ApJ, 653, L13

Weedman, D. W., et al. 2005, ApJ, 633, 706

Werner, M. W., et al. 2004, ApJS, 154, 1 